\title{
Archéopages
}

Archéopages

Archéologie et société

46 | 2018

Maisons

\section{La baraque militaire dans les hôpitaux américains de la Grande Guerre}

Stratégies économiques et choix socio-culturels

The military barracks in American hospitals of the Great War. Economic

Strategies and Socio-Cultural Choices

La caseta militar de los hospitales estadounidenses durante la Primera Guerra

Mundial. Estrategias económicas y elecciones socioculturales

Alexandre Coulaud, Robin Perarnau, avec la collaboration de Guillaume Charrière et Manon Mariette

\section{OpenEdition}

Journals

Édition électronique

URL : https://journals.openedition.org/archeopages/4355

DOI : 10.4000/archeopages.4355

ISSN : 2269-9872

Éditeur

INRAP - Institut national de recherches archéologiques préventives

Édition imprimée

Date de publication : 1 décembre 2018

Pagination : 80-85

ISSN : 1622-8545

Référence électronique

Alexandre Coulaud, Robin Perarnau, avec la collaboration de Guillaume Charrière et Manon Mariette,

"La baraque militaire dans les hôpitaux américains de la Grande Guerre ", Archéopages [En ligne], 46 | 2018, mis en ligne le 01 décembre 2020, consulté le 03 juin 2021. URL : http://

journals.openedition.org/archeopages/4355; DOI : https://doi.org/10.4000/archeopages.4355 


\author{
Alexandre Coulaud Inrap, Association franşaise de recherche en archéologie contemporaine (Afrac) \\ Robin Perarnau Arac \\ avec la collaboration de Guillaume Charrière Université de Bourgogne-Franche-Comté, Afrac \\ et Manon Mariette Universite de Bourgogne-Franche-Comté, Afrac
}

1. Provenant des forêts de pinèdes du sud des États-Unis.
Les récentes études archéologiques menées sur les hôpitaux construits par l'American Expeditionary Forces (AEF) (1917-1920) durant la Grande Guerre soulèvent de nombreuses interrogations. Comment une petite armée professionnelle d'à peine 100 ooo hommes a-t-elle pu se convertir en force militaire de premier plan et rendre opérationnels en seulement quelques mois des dizaines d'hôpitaux militaires à la pointe de la technologie? Ces immenses camps, édifiés en pleine campagne française, ont accueilli des dizaines de milliers de blessés venant du front avec une capacité totale de 200 ooo lits (Federal Reserve Bank of New York, 1919). Villeschampignons standardisées mais conçues selon une architecture modulaire, ils sont devenus le nerf de la stratégie militaire américaine et préfigurent les grandes opérations des $\mathrm{Xx}^{\mathrm{e}}$ et $\mathrm{XXI}^{\mathrm{e}}$ siècles. Ainsi, les infrastructures médicales (salles d'opération, morgues, laboratoires, etc.) et celles liées à d'autres activités (approvisionnement, loisir, stockage, etc.) sont toutes construites sur le modèle de la baraque militaire. Architecture nomade héritée des réflexions médicales, des conflits militaires et des grandes expéditions coloniales du XIX ${ }^{\mathrm{e}}$ siècle, les baraquements américains sont le reflet de pratiques sociales et culturelles. Ils constituent le lieu de vie du personnel soignant et des soldats blessés, leur maison, mais également un outil de reconquête de leurs pratiques socio-culturelles dans un pays qui leur est étranger.

\section{Une conception héritée $\mathrm{du} \mathrm{XIX}^{\mathrm{e}}$ siècle}

Bien que les hôpitaux de campagne existent à part entière depuis le XVIII ${ }^{\mathrm{e}}$ siècle sous leur forme moderne (en tant que service médical distinct), les premiers hôpitaux sous baraquement furent développés et expérimentés par les Britanniques et les Français lors de la Guerre de Crimée (18531856). Le développement de la médecine moderne apporte alors de nouvelles réflexions sur le traitement de masse des blessés civils et de guerre. Lobservation de «mauvaises effluves stagnantes » dans les hôpitaux de toile favorise la réalisation d'hôpitaux militaires sous baraquements de bois, où la circulation de l'air évite la transmission des fièvres et bactéries (Meynen, 2009). Ces réflexions vont logiquement influencer la manière de conceptualiser les locaux communs, sanitaires et administratifs ainsi que les logements des personnels soignants. L'architecture de la baraque tire aussi parti de l'expérience acquise lors des grandes expéditions coloniales du XIX ${ }^{\mathrm{e}}$ siècle (Fares, 2012).

Forts de ces observations, les architectes militaires du début du $\mathrm{XX}^{\mathrm{e}}$ siècle conçoivent les plans de baraques préfabriquées, aux dimensions standardisées, modulables et démontables. La réalisation de ce modèle hospitalier, construit en dur, hors d'eau et hors d'air comprenant chauffage, électricité et eau courante se généralise. Aux États-Unis, le développement du fordisme appuie cette conception architecturale, tout en permettant la mise en place de véritables chaînes de production. Dès 1917, chaque planche de bois ${ }^{1}$ est standardisée, découpée selon des dimensions précises, avant de servir à l'assemblage de panneaux qui seront expédiés en France [ill. 1]. Cette chaîne opératoire sera ensuite dupliquée dans l'Hexagone, le bois étant prélevé directement sur les forêts des Landes et des Vosges puis découpé grâce à l'installation de 81 scieries mécanisées (Perarnau, 2016). 
1.Plan d'assemblage et axonométrie d'une baraque américaine de type

« Burton-MacNeille »,1918 (ui Portable Houses for

Overseas Forces Built in

Record Time by New

Methods n, Engineering

News-Record, vol. 80, $n^{\circ} 1$,

1918, p. 40-42, dans

Perarnau, 2016).

2. En haut, baraque

démontable de type Aen bois

et soubassement en pierres

calcaires. Ce type de

baraquement, généralement préfabriqué et directement importé des États-Unis peut être construit en une journée par six hommes. En bas,

construction d'une baraque à ossature bois, fondations en pierres calcaires, appareil en briques et couverture en shingle (carton goudronné), Hospital Centerde Marssur-Allier, 1917-1919.
2. Diagnostic réalisé à Saint-Parize-le-Châtel / Magny-Cours (Nièvre) en juin 2014 sous

la direction de Nicolas Tisserand assisté d'Alexandre Coulaud. 3. Prospection pédestre réalisée en 2016 dans

le cadre d'un mémoire de Master II.

4. Prospection pédestre réalisée en 2017 dans le cadre d'un mémoire de Master II.
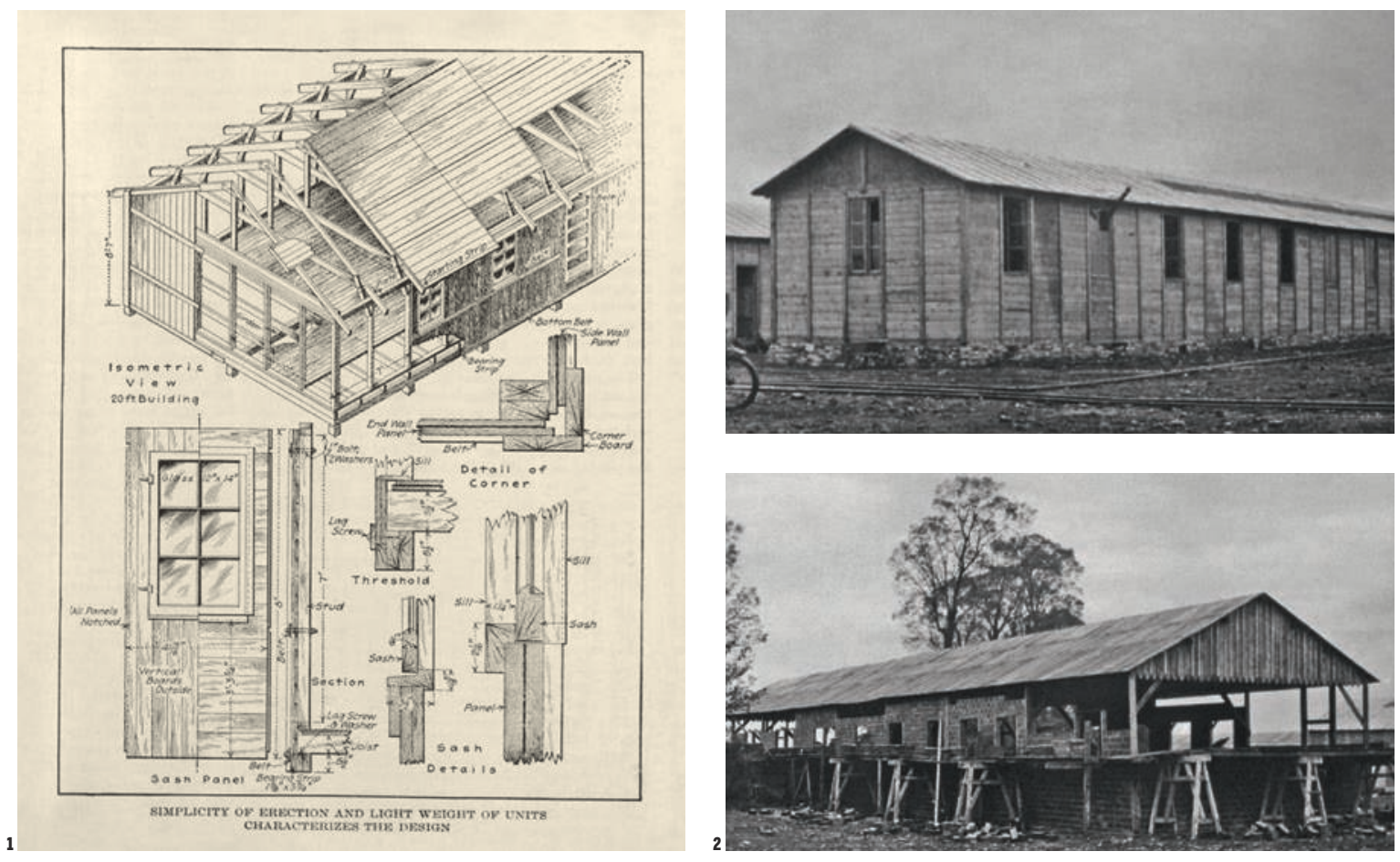

L'entrée en guerre des États-Unis, le 6 avril 1917, entraîne en effet un déploiement logistique colossal : 2 millions d'hommes, environ 600 infrastructures, plus de 200 sites à travers tout le territoire français et reliés entre eux par un nouveau réseau ferroviaire doublant le réseau existant. Ces scieries, carrières, usines, hôpitaux, dépôts de matériels et de munitions sont autant de signes de l'industrialisation massive des ÉtatsUnis. Jusqu'alors isolée du conflit, cette nouvelle puissance économique mondiale montre le déploiement extraordinaire dont elle est capable. De véritables villes à la pointe de la technologie voient le jour dans les campagnes françaises, jusqu'alors à l'écart des avancées scientifiques, techniques et technologiques modernes.

\section{Une architecture modulable}

Les recherches archéologiques menées ces dernières années confirment que l'utilisation de matériaux d'importation n'est pas systématique, les matériaux utilisés pour construire les baraques variant en fonction de leur disponibilité et de la proximité des sources de matières premières. On constate ainsi à Mars-sur-Allier ${ }^{2}$ (Nièvre) l'utilisation systématique de pierre calcaire, de briques et de béton d'origine locale pour les soubassements [ill. 2] (Coulaud et Tisserand, 2014, 2016, 2017 ; Mariette, 2017). À Beaune 3 (Côted'Or ; Perarnau, 2016) et à Mesves-Bulcy ${ }^{4}$ (Nièvre ; Charrière, 2017), si la pierre est remarquablement absente, de nombreuses dalles en béton servent, en revanche, de fondations [ill. 3]. En effet, la présence de gravières à proximité des deux sites permet d'obtenir du béton rapidement et en grande quantité. Les briqueteries françaises locales fournissent quant à elles l'essentiel de la production de terre cuite architecturale. Cette variation dans la provenance et l'utilisation des matériaux n'implique pas de différence architecturale majeure : la baraque américaine reste standardisée, hors-sol et hors d'eau tout en étant caractérisée par la présence d'une charpente en bois, et ce, quel que soit le site ou sa fonction. Le choix d'une forme de charpente préfabriquée et répétitive, et d'un assemblage par tenons et mortaises de fermes et de panneaux, permet de modifier les dimensions et donc les usages de la baraque militaire.

Les vestiges archéologiques montrent une relative similarité puisqu'on ne retrouve que les soubassements des structures. L'interprétation ne peut être établie que par la découverte de mobilier spécifique, par les dimensions du bâtiment ou bien par ses conditions d'implantation. Cette difficulté a été au cœur des problèmes soulevés lors des opérations archéologiques menées depuis 2011 sur les camps américains en France. Rien ne distingue en effet une dalle rectangulaire en béton de 1917 d'une autre, si ce n'est ses dimensions.

Par ailleurs, une baraque n'est pas une construction autonome, et n'a sa place que dans un ensemble plus vaste, le baraquement et le camp militaire. Son utilisation est donc liée à la présence d'autres structures, similaires ou non, à la disponibilité d'autres formes d'habitat et à la proximité d'autres infrastructures. Un hôpital américain (Hospital Center) est constitué de plusieurs unités hospitalières, appelées Base Hospital. Chacune de ces unités est construite selon un plan d'aménagement précis, et elles fonctionnent indépendamment l'une de l'autre comme autant d'hôpitaux distincts. Chaque base regroupe tous les services nécessaires à son 


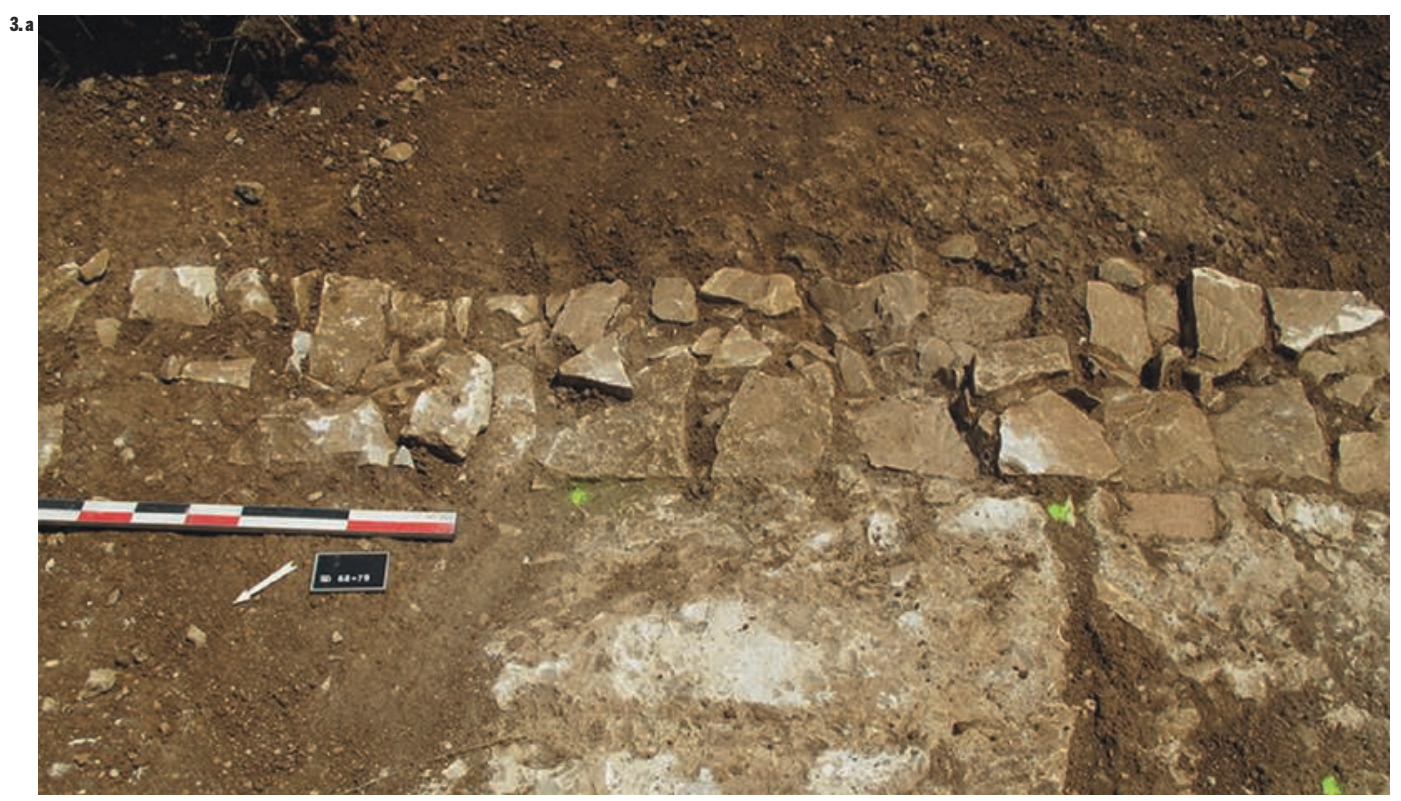

3. a. À l'Hospital Centerde Mars-sur-Allier (1917-1919),

l'utilisation conjointe

de béton (pour les sols)

et de pierres calcaires

(pour les murs de fondation)

est fréquente pour les

soubassements.

b. Vestiges de fondations

en béton d'un baraquement

de l'hôpital américain

de Beaune (1917-1920).

c. Vestiges de fondations en

briques et béton de l'Hospita

Centerde Mesves-Bulcy

(1917-1919).
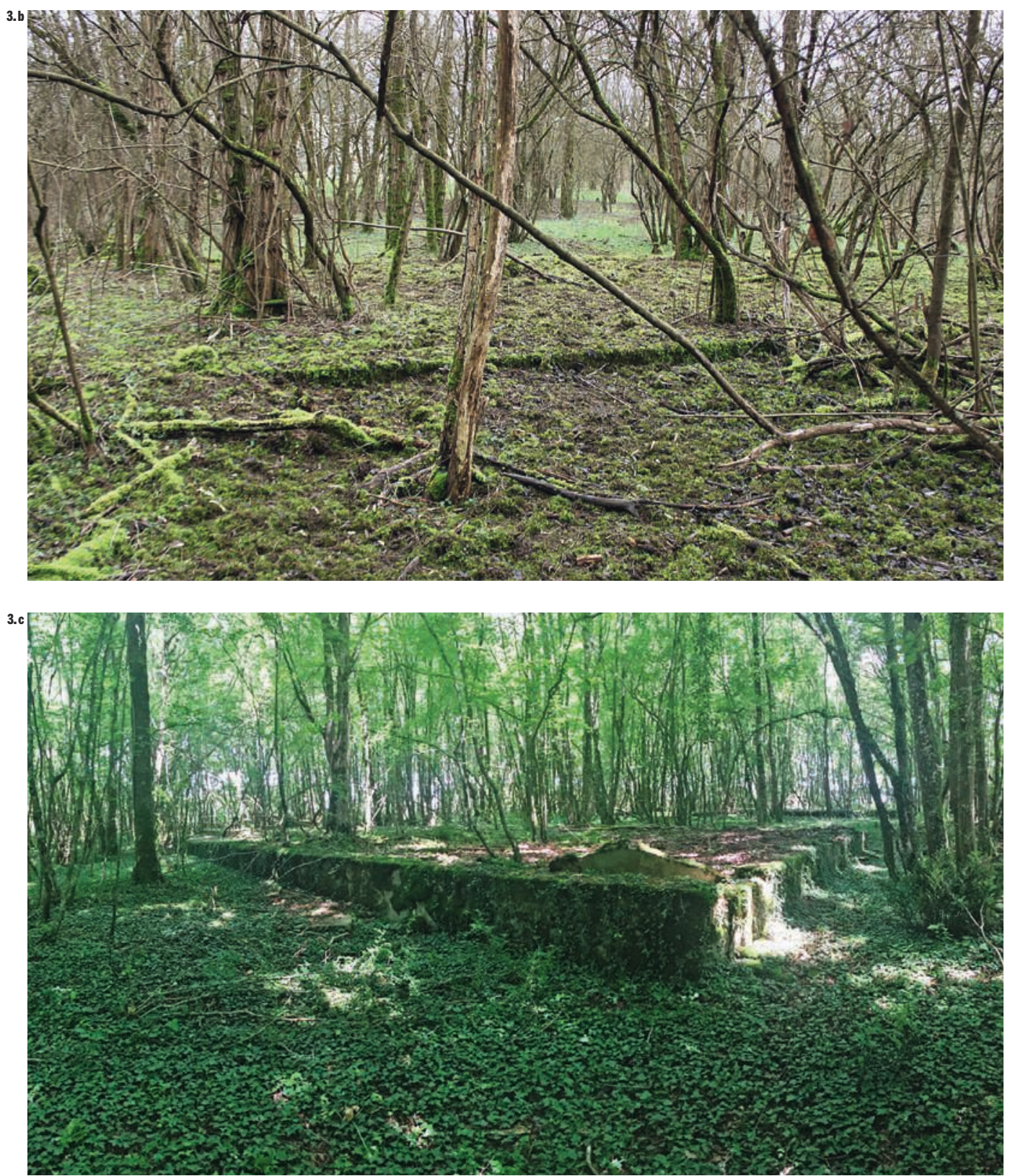
fonctionnement (administration, logistique, dortoirs, restauration, hygiène, services médicaux, etc.). Au sein du plan d'aménagement de chaque unité, tous les bâtiments ayant la même fonction ont des dimensions similaires. Ainsi, on retrouve sur les sites de Beaune, de Mars-sur-Allier et de Mesves-Bulcy des dalles de béton de 20 pieds $(6,10 \mathrm{~m})$ de large pour les fondations des dortoirs, montrant la similarité inter- et intra-sites des plans d'aménagements des Base Hospital.

Dans le contexte d'un plan d'aménagement strict, conçu et pensé en amont, comment l'architecture peut-elle s'adapter aux évolutions des besoins et des contraintes du conflit? La modification des baraques existantes ou la reconstruction de nouvelles structures en lieu et place des anciennes est impossible du fait de la présence des voies de circulation attenantes. La construction d'unités hospitalières supplémentaires nécessite donc la mise en œuvre d'un nouveau plan d'aménagement. Cette hypothèse est étayée par la présence de certains vestiges du camp de Mesves-Bulcy. Sur ce site, la dernière Base Hospital édifiée en urgence compte des dortoirs de $10 \mathrm{~m}$ de large, construits selon le même modèle que les baraques plus anciennes.

\section{L'espace domestique}

C'est l'aménagement intérieur qui transforme la baraque, espace neutre, en un lieu habitable ou utile à différentes fins. En cela, la baraque est une construction sociale, elle est le reflet de la volonté de ses occupants et de leurs pratiques socio-culturelles, autant que son architecture reflète les choix économiques des bâtisseurs. La transformation en espace domestique à destination des officiers et du personnel soignant est conçue de manière à faciliter leur installation et à leur garantir un confort optimal. D'après les plans du Medical Corps, les chambres sont individuelles ou doubles pour les infirmières [ill. 4] et les hauts-gradés, au contraire des dortoirs réservés aux officiers subalternes, aux soldats et aux patients. Ces partitions de bâtiment ne se repèrent pas en contexte archéologique, puisqu'elles sont généralement réalisées à l'aide de simples cloisons en panneaux de bois. Cependant, les archives iconographiques démontrent la présence de cuisines, salles de confort, et réfectoires directement installés au sein des baraques d'officiers et d'infirmières.

Au sein de l'American Expeditionary Forces et plus particulièrement du Medical Corps, la volonté d'installer les patients et le personnel dans les meilleures conditions possibles est flagrante : confort moderne, espaces de vie personnelle garantissant une part d'intimité pour les officiers et les infirmières, espaces de vie sociale [ill. 5]. Tout est mis en œuvre pour améliorer le moral des troupes, éloignées de leur pays d'origine et de leurs proches, confrontées à une guerre industrialisée et déshumanisée aux conséquences psychologiques terribles (morts de masse, amputations, utilisation des gaz de combat, épidémies). L'organisation d'activités collectives au sein des foyers permet d'entretenir une forme de cohésion de groupe au sein du personnel, nécessaire au bon fonctionnement du camp. La structure militaire américaine reste néanmoins très hiérarchisée et chaque catégorie de personnel dispose de ses propres quartiers dédiés.

\section{Un reflet des pratiques socio-culturelles}

Les Américains construisent des infrastructures spécifiques et importent quantité de matériel à caractère domestique, dont des traces subsistent dans les archives, dans l'iconographie et en contexte archéologique. Les événements organisés dans le camp et pléthore d'objets de la vie quotidienne retrouvés sur le terrain constituent le reflet de pratiques socio-culturelles américaines. Dans les baraques ou dans des immenses hangars de type Bessonneau ${ }^{5}$, on organise des événements sportifs (baseball, football américain, basketball) et culturels (arrivée du jazz en Europe, concerts, spectacles, théâtre, bibliothèques, diffusion d'un journal du camp). La structure militaire américaine facilite également l'insertion d'associations non gouvernementales dédiées au confort et à l'animation auprès des soldats et du personnel, American Red Cross et YMCA (Young Men's Christian Association) notamment (Orr, 1919).

Espaces domestiques de substitution, les logements du personnel ainsi que les foyers accueillent les activités quotidiennes que les Américains pratiquaient à leur domicile. Létude des archives révèle que beaucoup d'éléments matériels civils ont été importés depuis les ÉtatsUnis. Après le départ des Américains aux débuts des années 1920, les très importants stocks d'objets de l'American way of life que les États-Unis ne peuvent pas rapatrier (Pershing, 1920) sont cédés à la France pour des sommes modiques, puis mis en circulation sur le marché via le concours de coopératives et d'entreprises privées. C'est ainsi qu'un grand nombre de foyers français font la découverte de toute une série d'objets et de denrées alimentaires : brosse à dents en plastique (celles-ci sont encore en os en France), corned beef, bacon, etc. Le mobilier mis au jour lors des récentes opérations archéologiques à Mars-surAllier (Nièvre), Is-sur-Tille ${ }^{6}$ (Côte-d'Or) (Devevey, 2011), Mesves-Bulcy (Nièvre) et Beaune (Côted'Or) fournit toute une série d'exemples : rasoir Penn, brosse à dents Prophilactic, paquets de tabac Prince Albert, vaisselles, nécessaires de couture, journaux, habillement civil, parfums, produits d'hygiène, etc. Autant de marchandises que les soldats et personnels américains ont apporté avec eux ou qu'ils peuvent acheter sur place, en France, dans les boutiques des différents camps gérées par l'armée américaine, leur permettant ainsi de continuer à consommer leurs produits du pour l'aviation civile et militaire.

6. Sondages menés en 2011 sous la direction $\mathrm{d}$ Frédéric Devevey, Inrap. 

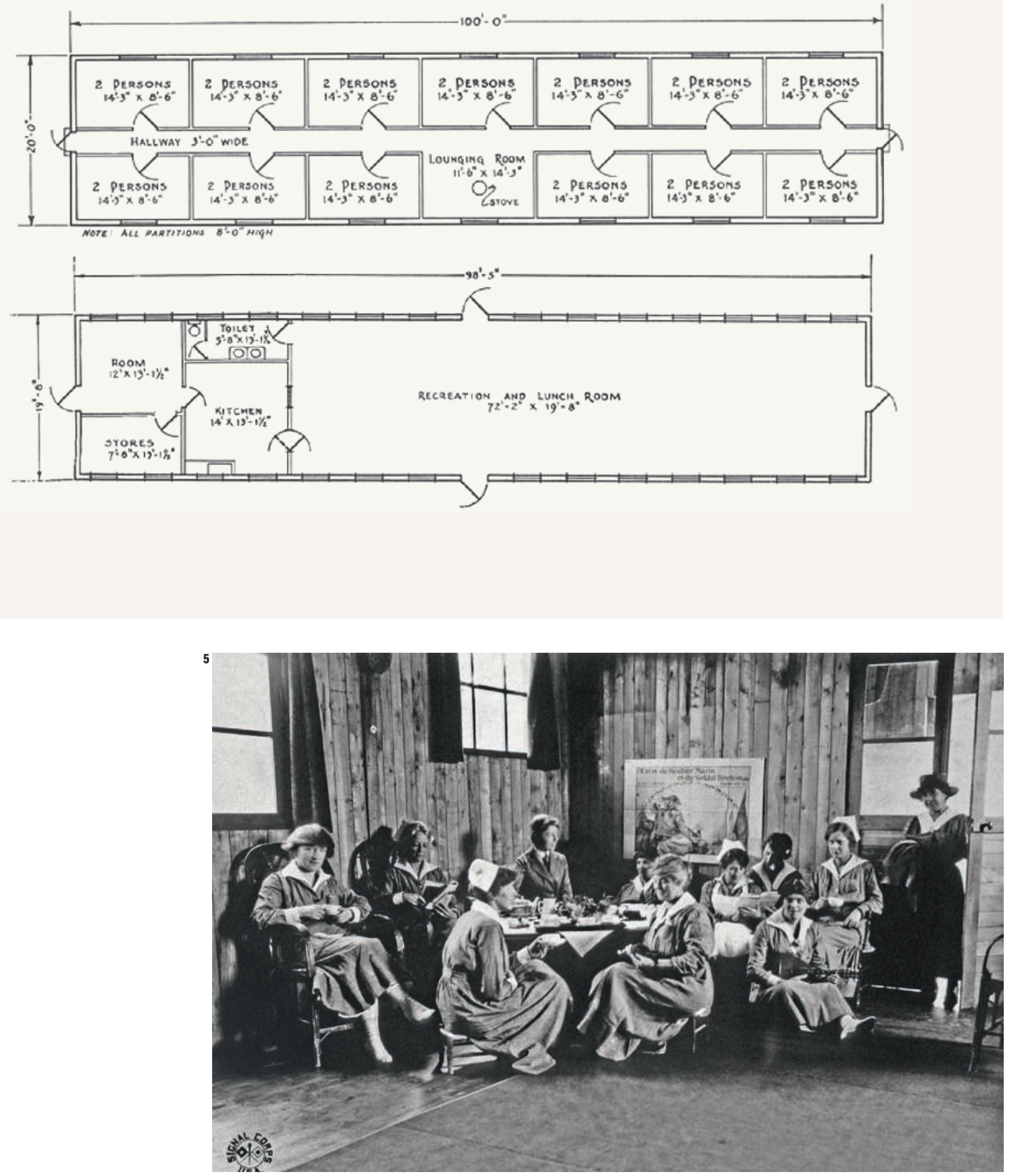

4. Plans des baraques destinées à accueillir les chambres (en haut) et le foyer des infirmières (en bas). II s'agit de baraques démontables en bois de type $A$, généralement préfabriquées et

majoritairement importées en pièces détachées des États-Unis (Ford, 1927).
5. Scène de repos, foyer des infirmières, Base Hospita $n^{\circ}$ 17, Dijon (1917-1919). Discussions, lectures, couture autour d'un thé chez ces infirmières dans un cadre chaleureux avec une décoration personnelle qu n'est pas sans rappeler les images de l'intérieur des maisons de l'Ouest américain de la fin du XIXe siècle.
6. Vue de l'intérieur d'une chambre d'infirmière, Base Hospital n 58, Mars-surAllier. La décoration y est très fournie et personnelle: photographies, miroirs,

tissus, dessins, rubans, etc. 


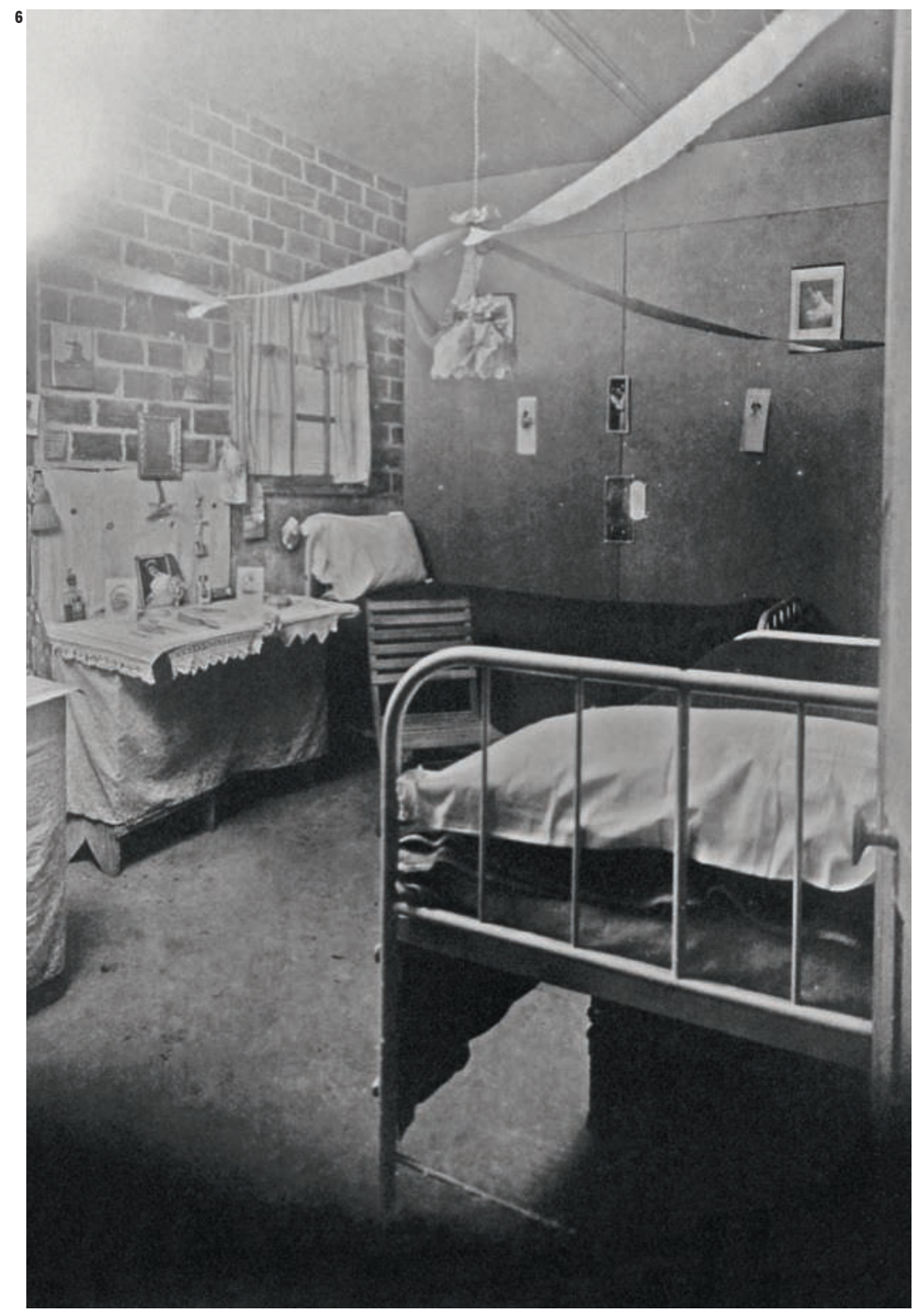

quotidien. Ce phénomène de consommation « militaire » est d'ailleurs toujours d'actualité dans les énormes PX (Post Exchange) de l'armée américaine en Afghanistan ou en Irak.

Par ailleurs, certaines catégories de mobilier comme la vaisselle céramique dénotent un certain luxe, relativement rare dans ce contexte de conflit où l'on est plutôt habitué à utiliser le quart et la gamelle en aluminium, mais cohérent dans le cadre d'un hôpital de campagne muni d'espaces de détente. La décoration parfois très personnelle est perceptible sur certains clichés d'époque des chambres [ill. 6] et des foyers.

La baraque militaire convertie en espace domestique est soumise à un ensemble de règles et de choix stratégiques. Cet habitat, loin d'être improvisé, est en réalité le fruit d'une réflexion médicale construite au $\mathrm{XIX}^{\mathrm{e}}$ siècle, ainsi que d'une logique militaire, économique et logistique. Malgré cette conception en amont, les baraques américaines s'adaptent aux conditions d'implantation du site, aux matériaux disponibles et à la fonction du baraquement. Cette architecture modulaire, en pièces préfabriquées, permet donc de répondre aux besoins et aux contraintes militaires. L'identification des structures d'habitat parmi les vestiges archéologiques n'est pas aisée. La comparaison des archives textuelles et iconographiques avec les données archéologiques permet cependant de formuler des interprétations sur la place de la sphère domestique dans le contexte d'un hôpital militaire. Plus qu'une simple structure, le baraquement américain apparaît comme le reflet de la culture socio-économique américaine et comme un lieu domestique de substitution, permettant détente, intimité, cohésion et confort, aux soldats et aux personnels loin de leurs attaches.

\section{Références bibliographiques}

ANONYME, 1918, « Portable Houses for Overseas Forces Built in Record Time by New Methods ", Engineering News-Record, vol. 8o, n ${ }^{\circ}$, p. 40-42.

Charrière G., 2017, Étude du réseau hospitalier américain de la Première Guerre mondiale : l'exemple du camp de Mesves dans la Nièvre, Mémoire de Master II AGES, Dijon, Université de Bourgogne-Franche-Comté, 81 p. + annexes.

Coulaud A., Tisserand N., 2014, Saint-Parize-leChâtel, Magny-Cours, les Cômes, les

Viollettes : occupation néolithique et témoignage exceptionnel de la première guerre mondiale: l'Hôpital américain de Saint-Parize-le-Châtel, rapport d'opération, vol. 1, Inrap-SRA Bourgogne, $97 \mathrm{p}$.

Coulaud A., Tisserand N., 2016, «L'Hospital Center de Mars-sur-Allier dans la Nièvre, un gigantesque complexe », Archéologia, 548, p. 44-45.
Coulaud A., Tisserand N., 2017, « Organisation médicale pendant la Première Guerre mondiale, l'exemple de l'hôpital américain de St-Parize-leChâtel », Archéopages, 43, Médecines, p. 68-71.

Devevey F., 2011, Marcilly-sur-Tille (Côte-d'Or), la Combotte-Champs Blancs : sondages archéologiques sur la gare régulatrice française, 1914-1919, de Marcilly-sur-Tille, rapport d'opération, Inrap-SRA Bourgogne, $102 \mathrm{p}$.

FARES K., 2012, L'industrialisation du logement en France (1885-1970): de la construction légère et démontable à la construction lourde et architecturale, Thèse de Doctorat du Conservatoire national des arts et métiers, Histoire des Techniques et de l'Environnement, $518 \mathrm{p}$.

ForD J. H., 1927, The medical department of the United State army in the World War, vol. II, Administration American Expeditionary Forces, United States Government Printing Office, $1137 \mathrm{p}$.

Federal Reserve Bank Of New York, 1919, Facts about the way the United States made war, New York, Governement Loan Organization, 24 p.
MEYNEN N., 2009, «Les hôpitaux militaires sous tentes et baraqués au XIX ${ }^{\mathrm{e}}$ siècle », Revue historique des armées, 254, p. 92-109.

Mariette M., 2017, Approche archéologique d'un site contemporain : l'hôpital militaire américain de Mars-sur-Allier (Saint-Parize-le-Châtel, Nièvre), Mémoire de Master I AGES, Dijon, Université de Bourgogne-Franche-Comté, 50 p. + annexes.

OrR W., 1919, Educational work of YMCA, 1916-1918 Washington Government Printing Office, 6o p.

Perarnau R., 2016, Contribution à l'étude archéologique de la logistique américaine durant la première guerre mondiale à travers l'exemple du camp américain de Beaune (1918-1919), Mémoire de Master II AGES, Dijon, Université de Bourgogne, 72 p. + annexes.

Pershing J. J., 1920, Final Report of Gen. John J. Pershing, Commander-in-Chief American Expeditionary Forces, Washington, Government Printing Office, 180 p. 